
2021
Revista Vol. 24(47)
Prolegómenos enero-junio - ISSN: 0121-182x - e-ISSN: 1909-7727 - pp. 39-54

DOI: https://doi.org/10.18359/prole.4611

\title{
Propuesta de deconstrucción del discurso del desarrollo y alternativas basadas en la sustentabilidad*
}

\author{
Paula Nathalia Correal Torres ${ }^{a}$. Nohemí Bello Gallardo ${ }^{b}$
}

\begin{abstract}
Resumen: el artículo presenta un análisis crítico en torno al discurso del desarrollo, desde la Antigüedad clásica hasta el siglo XXI, y su papel frente a la preservación ambiental. A fin de establecer la relación entre progreso y medioambiente, se abordaron temáticas como la sustentabilidad y posibles alternativas a esta, que se construyen, desde la ciencia y la política, para responder a una tendencia de mercantilización de la naturaleza, incorporando elementos como la racionalidad ambiental, la ley límite de la naturaleza y la ecología política. En este aspecto, resulta apropiado revisar el papel de intelectuales y científicos que han abordado el tema, especialmente, en la inclusión de los aspectos sociales y ambientales dentro del núcleo semántico del desarrollo. El estudio se llevó a cabo bajo un método analítico, de investigación básica, de enfoque cualitativo y descriptivo, cuyas fuentes son documentales.
\end{abstract}

Palabras clave: ciencia y poder; desarrollo; intelectuales orgánicos; mercantilización de la naturaleza;

sustentabilidad; visión biocéntrica

Recibido: 19 de febrero de 2020

Aceptado: 23 de febrero de 2021

Disponible en línea: 06 de agosto de 2021

Cómo citar: Correal Torres, P. N., \& Bello Gallardo, N. (2021). Propuesta de deconstrucción del discurso del desarrollo y alternativas basadas en la sustentabilidad. Prolegómenos, 24(47), 39-54. https://doi.org/10.18359/prole.4611

* El presente artículo de reflexión corresponde a los hallazgos del proyecto de tesis doctoral Análisis de los megaproyectos desde la teoría de la subalternidad y la autonomía en México, desarrollado en la Universidad Autónoma de Querétaro, Doctorado en Ciencias Jurídicas.

a Maestra en Justicia Constitucional. Universidad Autónoma de Querétaro, Querétaro, México. Correo electrónico: paula.correal.torres@gmail.com ORCID: https://orcid.org/0000-0003-4114-0280

b Doctora en Derecho Público. Universidad Autónoma de Querétaro, Querétaro, México. Correo electrónico: dra.nohemibg@gmail.com ORCID: https://orcid.org/0000-0002-6916-7816 


\title{
Proposal for the Deconstruction of the Development Discourse and Sustainability-Based Alternatives
}

\begin{abstract}
: the article presents a critical analysis of the development discourse, from classical antiquity to the 21st century, and its role in environmental preservation. In order to establish the relationship between progress and environment, issues such as sustainability and possible alternatives to it were addressed. Those alternatives are built up from scientific and political knowledge to respond a trend of commodification of nature incorporating elements such as rationality, the limiting law of nature and political ecology. In this regard, it is appropriate to review the role of intellectuals and scientists who have tackled this issue, especially in the inclusion of social and environmental aspects within the semantic core of development. The study was carried out by an analytical basic resarch method with a qualitative and descriptive approach, which sources are documentary.
\end{abstract}

Keywords: science and power; development; organic intellectuals; commodification of nature; sustainability, biocentric perspective

\section{Proposta de desconstrução do discurso do desenvolvimento e alternativas baseadas na sustentabilidade}

Resumo: neste artigo, apresenta-se uma análise crítica sobre o discurso do desenvolvimento, desde a Antiguidade clássica até o século xxi, e seu papel ante a preservação ambiental. A fim de estabelecer a relação entre progresso e meio ambiente, foram abordadas temáticas como a sustentabilidade e possibilidades alternativas a esta, que são construídas, a partir da ciência e da política, para responder a uma tendência de mercantilização da natureza, incorporando elementos como a racionalidade ambiental, a lei-limite da natureza e a ecologia política. Nesse aspecto, resulta apropriado averiguar o papel de intelectuais e cientistas que abordam o tema, em especial na inclusão dos aspectos sociais e ambientais dentro do núcleo semântico do desenvolvimento. Este estudo foi realizado com base num método analítico, de pesquisa básica, de abordagem qualitativa e descritiva, cujas fontes são documentais.

Palavras-chave: ciência e poder; desenvolvimento; intelectuais orgânicos; mercantilização da natureza; sustentabilidade, visão biocêntrica 


\section{Introducción}

El concepto de desarrollo, como discurso de cosificación del mundo, circunscrito tendencialmente a la esfera económica, tiene su ejemplificación clara en las modificaciones al Fondo Monetario Internacional (FMI). Estas modificaciones surgen de las relaciones de poder entre los países llamados desarrollados o de primer mundo, y las economías emergentes o países en vías de desarrollo. Se menciona esta situación para ubicar el contexto al que el artículo se circunscribe, toda vez que estas clasificaciones y su modificación obedecen al aumento del producto interno bruto (PIB) de cada país, para ubicar su posición en el extremo desarrollado o en vías de desarrollo.

Las organizaciones globales de geopolítica se desenvuelven bajo criterios económicos, que dan poder a países con mayor PIB sobre las decisiones que afectan a toda la humanidad, como es el caso del veto de decisiones tomadas en acuerdo multilateral (Torres, 2008). De acuerdo con estos movimientos de poder, basados en el discurso del desarrollo, es importante analizar su impacto en temáticas como la protección medioambiental, a fin de determinar si esta se ve realmente ralentizada o las dinámicas de mercado y presiones mediáticas hacen que se convierta o se encamine a convertirse en prioridad para individuos y Estados.

Las dinámicas de los gobiernos y las campañas políticas de cualquier corriente que presentan el desarrollo como promesas basadas en el aumento del ingreso per cápita, la libertad de acción de las grandes empresas y la idea de bienestar económico de la población, dejan en último lugar al medioambiente o, incluso, lo excluyen del panorama de avance nacional. Los recursos naturales se reducen a materia dominada por el hombre, su utilidad en la industria y su transformación.

Las cortapisas frente a las acciones globales para proteger el medioambiente son numerosas; los retiros de acuerdos internacionales o su no participación marcan un camino accidentado hacia la preservación de los recursos naturales, toda vez que el incumplimiento de los compromisos o la ausencia de acuerdo en puntos clave generan consecuencias que van desde la desconfianza en este tipo de acuerdos multilaterales hasta el descrédito de las afirmaciones surgidas de allí. Tal fue el caso del aplazamiento de la discusión sobre la reducción de la contaminación global del aire y la reglas para los mecanismos del mercado voluntario, en la Conferencia de las Partes de la Convención sobre el Cambio Climático (COP) 24 (Murat, 2018).

Ahora bien, estas decisiones que impactan globalmente se fundamentan en estudios económicos, políticos y, en general, científicos, que se presentan de acuerdo con los extremos y los intereses que defienden. De esta manera, organizaciones ambientalistas como Greenpeace llegan a descartar los efectos negativos del cambio climático para justificar la negativa a programas nucleares, de los cuales se piensa que pueden mitigar los efectos del calentamiento global al no producir gases de efecto invernadero (GEI). Mientras que, en el otro extremo, se ubican los dueños de grandes empresas dedicadas a los combustibles fósiles, que niegan rotundamente la existencia del cambio climático.

De esta manera se orienta la labor científica y la producción del conocimiento hacia la defensa de intereses económicos. Como ejemplos de ello, pueden observarse los estudios que plantean las ventajas del fracking, la activación de programas de energía basados en combustibles fósiles, la negación del calentamiento global, las ventajas de no pertenecer a organizaciones multilaterales y legitimar el uso indiscriminado y depredador de los recursos naturales, entre otros. Los casos donde se ve reflejada esta situación de conducción científica hacia intereses determinados, se explica en el segundo apartado de la investigación, con ejemplos como el desastre del municipio de Seveso (Italia), el plan de energía norteamericano y las iniciativas de firma contra las acciones de mitigación del cambio climático.

Actualmente, están planteándose alternativas frente al problema más difícil y vigente: la supervivencia, problema que afecta a toda la humanidad y exige reconsiderar las prioridades. Los efectos pueden verse a partir de fenómenos como el aumento del nivel del mar, el deshielo de los polos, los problemas de salud que enfrenta la población por la baja calidad del aire, la contaminación de fuentes hídricas y las sequías que amenazan la 
producción de alimentos, entre otros. Las causas de estos fenómenos no obedecen del todo a causas antropogénicas, pero la mano del hombre sí produce un aumento en la variabilidad climática y la reducción de la periodicidad de las variaciones. Este aspecto se amplía al analizar la teoría de la sustentabilidad.

La conservación del medioambiente y los recursos naturales es garantía para la supervivencia humana. Las condiciones de la biósfera y la preservación del equilibrio ecosistémico son indispensables para la prolongación de la vida humana y sus futuras generaciones. La preocupación internacional por esta cuestión, unida a la preservación ambiental se materializa en el informe de Brundtland (1987), realizado para la Organización de Naciones Unidas por la ex primera ministra noruega Harlem Brundtland, con el fin de analizar el desarrollo económico y contrastarlo con la idea de la sustentabilidad, teniendo en cuenta los costos ambientales del crecimiento económico. Si bien este instrumento resulta relevante como primer acercamiento a nivel internacional sobre la preocupación por el medioambiente, las medidas tomadas para la conservación ambiental han sido mínimas e infructuosas.

La sustentabilidad, en tanto concepto amplio e indeterminado, requiere replantearse, para incluir elementos como la ley límite de la naturaleza. La idea consumista de recursos puede contrarrestarse a través de la ecología política, presentada por Enrique Leff y otros teóricos, cuyos aportes se reflejan en el apartado de alternativas al desarrollo, basadas esencialmente en tres pilares: (1) "la resistencia al neoliberalismo", (2) "la compensación por daños ecológicos" y (3) "la justicia ambiental" (Leff, 2006, p. 23).

\section{Breve comentario al nacimiento y evolución del discurso del desarrollo}

El término desarrollo se vincula al concepto de progreso. Este último se refiere al crecimiento o avance que la humanidad se traza como objetivo para perfeccionarse. Sin embargo, este concepto tiene pluralidad de interpretaciones, elementos y estrategias para definirlo. Su surgimiento puede ubicarse en la Edad Antigua, bajo la idea de avanzar, que se da desde el plano espiritual hasta el material. La idea principal del progreso en aquella época se circunscribía a la protección de la virtud moral. Ciertamente, el progreso bajo el significado de perfeccionamiento de la humanidad centraba su atención en el aspecto espiritual.

Algunas apreciaciones de la Antigüedad clásica dan cuenta de la caracterización del progreso, como la capacidad humana para avanzar constantemente, cambiar y adaptarse a las exigencias del entorno y la vida colectiva. Aunque logran vislumbrarse posiciones encontradas, puesto que la conservación de la virtud en algunos filósofos se pierde con la expansión del conocimiento; mientras que, en otros, se avanza hacia la perfección de la humanidad. No obstante, el punto medular al respecto es el incontenible avance del ser humano, para modificar sus condiciones de vida y su entorno, su capacidad tanto positiva como negativa de transformar la naturaleza.

Averroes (1126-1198), conocido comentarista de Aristóteles (384-322 a. C), planteó su visión del progreso a través de la ciencia, la razón y el saber. En sus comentarios a la Metafísica II, según señalan Rafael Ramón Guerrero (2005, pp. 13-28), Averroes circunscribió el progreso a la tradición y a la mejora, en el sentido de avance en el conocimiento, fundamentado en las siguientes premisas:

- El deseo innato del hombre de la verdad y los límites del intelecto para conocer las cosas inteligibles.

- El papel o contribución de los predecesores con sus comentarios o aclaraciones, unidos al carácter progresivo de la razón humana.

- La filosofía como ciencia de la verdad que investiga la realidad completa.

- La verdad como conocimiento de la causa.

La idea de Averroes fue retomada por Santo Tomás de Aquino (1225-1274), quien señaló que el progreso se da por las aportaciones de los predecesores, unidas a los aportes del individuo que las recibe. El progreso de la razón y el conocimiento de las cosas van unidas a la capacidad crítica, que 
permite recorrer el camino hacia el perfeccionamiento constante entre generaciones.

En sentido teológico, en La ciudad de Dios (2011), San Agustín (354-430) señaló que el progreso de la humanidad se realiza encaminado hacia la ciudad celeste, dejando atrás la ciudad terrenal. Lo que indica el obispo de Hipona es la búsqueda del progreso espiritual y no el material, pues este último impide conocer y ver a Dios. El progreso dado por el mal uso del libre albedrío resulta en una calamidad, en un principio depravado, que aleja a la humanidad de su fin último, que es agradar a Dios y lograr la construcción de una ciudad celeste.

Por su parte, Santo Tomás plantea en la Suma de teología (2001) que el progreso, el avance y en si el actuar humano, se encamina hacia un fin: la bienaventuranza. El ser humano como criatura racional, con sus fuerzas limitadas, no puede progresar, por lo cual requiere dirección de Dios. Asimismo, indica que la vida terrena tiene como fin que el alma progrese en conocimiento y mérito. Es decir, de Aquino divide en dos tipos el progreso que puede alcanzar el hombre: en gracia (mérito) y en ciencia (conocimiento de las cosas); ambos siempre mediados por la mano de un ser superior que revela verdades y otorga gracia. Enmarca el concepto de progreso como el camino de la imperfección hacia la perfección, por medio del conocimiento de la verdad, en los términos indicados en líneas anteriores.

San Anselmo (1033-1109), en su visión del progreso basado en la comprensión de Dios y la Escritura, conmina a ver la inteligencia como el término medio entre fe y visión. Su idea central, expuesta por André Hubert (2004), es progresar hacia la comprensión, por medio de la fe, y acercarse a la visión beatífica, con la gracia otorgada por Dios. Un elemento interesante al respecto es la idea de comunicación de esta comprensión a los demás, es decir, una vez se consigue progresar a nivel individual, debe compartirse este progreso, para que todos puedan caminar hacia la visión beatífica.

Al dejar de lado la concepción metafísica del ser humano, cuyo avance se presenta por designios de Dios, con lo que se impide la degeneración del ser, se vincula al término progreso con diferentes aspectos, como aquella organización social civilizatoria, de mejora o avance. También en Kant (1724-1804) se puede encontrar la vinculación del progreso con la deseable realización de un plan de la naturaleza, cuando indica que la historia de la humanidad puede considerarse "la ejecución de un plan oculto de la Naturaleza para llevar a cabo una constitución interior y - a tal fin- exteriormente perfecta, como el único estado en el que puede desarrollar plenamente todas sus disposiciones en la humanidad" (2006, p. 57).

Esta postura kantiana debe considerarse el deber ser. Puede observarse que la conexión del progreso o desarrollo del hombre con un plan de la naturaleza se basa en un movimiento a la acción. Ello significa que, al incluir aspectos como las facultades biológicas, el impulso de la colectividad y la cultura en la construcción de la sociedad cosmopolita y del individuo, estos desarrollarían sus capacidades, acorde a sus fines (Keferstein, 2005, p. 6).

El progreso es también relacionado con la riqueza por autores como Adam Smith (1776, p. 17), quien encontraba las causas del progreso en las capacidades productivas y la forma de distribución; progreso entendido como el disfrute de ciertas cosas (necesarias y cómodas) que mejoran la calidad de vida. Asimismo, John Stuart Mill (1984, p. 16) planteó la idea de progreso bajo el concepto de utilidad, es decir, la búsqueda de la felicidad del individuo a través de la posesión de bienes.

La naturaleza progresista de la raza humana fue estudiada por diferentes teóricos durante los siglos XVII-XIX, que aportaban elementos destacados del avance del ser humano hacia mejores condiciones de vida, desde posesión hasta ascensión a niveles culturales y morales elevados. A pesar de ello, durante el siglo XIX surgieron posturas escépticas sobre el progreso en la escena natural y social. Estas posturas marcaron la desmitificación del progreso siempre como avance hacia la mejora, o como curso natural de la esencia humana y su búsqueda de perfección. Bajo la óptica escéptica, el progreso se circunscribe a la búsqueda de relaciones causales que, en sí mismas, no son buenas o malas, ni obedecen al perfeccionamiento humano, sino a la búsqueda de la comprensión del mundo.

La construcción del discurso del desarrollo, como un fin deseable y deseado por todas 
sociedades, encuentra eco en su visión como contrapeso de la barbarie (Nisbet, 1979, p. 16), es decir, cuanto mayor es el progreso menor resulta la violencia. Además de presentarse como elevación y perfección de la raza humana, lo que se traduciría, a su vez, en mejores condiciones de vida, comodidad, cultura y felicidad. Pese a ello, en el siglo Xx, con los postulados escépticos incipientes de finales del XIX, la idea de progreso se vinculó estrechamente a la raza y la potencialidad económica.

La tendencia hacia el desarrollo, como mejor estado de cosas, se vio puesta a prueba en el siglo $\mathrm{xx}$ por las guerras, el deterioro medioambiental, la pérdida de recursos y la depresión económica. No obstante, la confianza puesta en el progreso como sinónimo de desarrollo logra evidenciarse en postulados que pronosticaban una elevación cultural, lo que permitiría una mejora de la humanidad. La idea de elevación y de continuidad del desarrollo se cimienta en la proliferación de avances industriales, técnicos, culturales y sociales, en la medida en que las consecuencias o efectos de la posguerra ponen a la humanidad en una unión momentánea (por el bien común).

Los vencedores de la Segunda Guerra Mundial avanzaron y se posicionaron en la cúspide de la pirámide del desarrollo. Especialmente, para Estados Unidos, se hizo tangible el discurso del desarrollo basado en la justicia, el aumento del capital y la supuesta cooperación con los denominados "subdesarrollados" por el expresidente Truman, en su discurso de posesión en 1949 (Esteva, 2000).

Este aspecto resulta central para comprender el anclaje del discurso del desarrollo a los procesos de avance en las sociedades. Para intuir la dinámica de medición del crecimiento con base en la evolución industrial y la cooperación entre países, resulta necesario conocer la interpretación de un economista como Angus Maddison (2004). Este autor ofrece un esquema claro y cuantitativo de la cuestión en la denominada "época de oro", que coincide con el punto cúspide de crecimiento del PIB en las grandes regiones mundiales. Es decir, el crecimiento económico se circunscribe a la idea de desarrollo.

En las últimas cuatro décadas del siglo xx, el deterioro ambiental pone en el panorama la evolución del concepto de desarrollo a desarrollo sustentable. Sin embargo, antes de abordar lo correspondiente a la sustentabilidad, es necesario mencionar a su antecesor, el ecodesarrollo, que apareció en escena en 1974 en la conferencia de Cocoyoc, como un conjunto de estrategias deseables y racionales desde el punto de vista económico, social y ecológico (Declaración de Cocoyoc, 1978, art. 4).

El concepto de "desarrollo sustentable" apareció en el escenario internacional en 1987, a partir de la preocupación surgida en 1960 por la pérdida de los recursos naturales, el desequilibrio social y el riesgo de la garantía de supervivencia humana, entendida esta última como la protección del medioambiente. La máxima que se defiende es la garantía de satisfacción de las necesidades presentes, sin comprometer las capacidades de supervivencia de las generaciones futuras. Desde la construcción de este concepto, se ha propendido por su apropiación y la generación de elementos comunes que permitan identificar este tipo de desarrollo. Muestra de ello fue la Cumbre de la Tierra en 1992, de la cual se derivaron instrumentos como las Convenciones sobre Cambio Climático y Biodiversidad, la Declaración de los principios de manejo, conservación y desarrollo sustentable y la Agenda 21.

En el inicio del siglo XxI, con la globalización en un punto acelerado y permeado en pluralidad de aspectos de la vida humana (comunicaciones, educación, tecnología, etc.), se hacen evidentes los problemas de un concepto amplio e indeterminado como desarrollo sustentable. La vinculación del discurso del desarrollo se enmarca en diferentes áreas, con preeminencia de la económica, pero ahora tratando de migrar hacia el pasado, con conceptos como el "buen vivir" o actualizar el desarrollo con movimientos como la globalización contrahegemónica como alternativas de pensamiento. 


\section{Conocimiento científico: instrumento de sustento del discurso del desarrollo}

Entendiendo por conocimiento una creencia correcta y justificada del sujeto, definición planteada por Andrew Brook y Robert Stainton (2000, p. 3) e incorporando la objetividad y la criticidad como características centrales, logra identificarse la imagen de la ciencia y el papel del científico, como agente de la verdad, es decir de la objetividad que va en contra de la visión escéptica. Esta visión rechaza la autoridad del pensamiento no propio del sujeto y propugna por un acercamiento al objeto de estudio de forma autónoma, obedeciendo solo a los propios convencimientos.

El conocimiento, en general, se plantea en términos de verdad. Sin embargo, el cientifismo se ve envuelto en una fe ciega desde y hacia la ciencia, olvidando que el concepto de verdad puede transformarse en una invención humana que facilite la convivencia social.

El conocimiento denominado científico no es ajeno a los andamiajes de poder; incluso la experimentación puede llegar a verse, cuanto menos, encauzada hacia la comprobación de teorías propias de ciertos sectores económicos. El papel del científico (intelectual) en la época actual requiere de un fuerte componente ético e, incluso, la misma ciencia debe estar comprometida con la objetividad. En efecto, la universalización de intereses y valoraciones se ve permeada por las normas y valores reconocidos intersubjetivamente en determinadas circunstancias (Habermas, 1990, pp. 2-5), haciendo del conocimiento presentado como científico, sin serlo, un paso de validación de verdades dadas por sentado previamente.

Este punto se encuentra en postulados de Marx en la crítica de la economía política, cuando se indica que la base del conocimiento es económica, haciendo de la síntesis un escenario de producción material, lo cual se contrapone al verdadero carácter del científico y la ciencia (Marx, 2008, p. 335). Es relevante mencionar que la objetividad que se exige del conocimiento científico se basa en la autonomía del científico, quien debe hacer a un lado los marcados intereses económicos que pretenden guiar su labor y centrarse en la búsqueda de la verdad.

La pertenencia a instituciones, centros de investigación o universidades reduce el campo de acción e investigación, de acuerdo con las áreas de pertinencia definidas en los diversos programas y planes de trabajo, para los cuales son contratados científicos e investigadores. Sin embargo, este aspecto no puede reducir a los científicos a un rol de intelectuales orgánicos, precisamente, por la naturaleza de la ciencia.

El papel de algunos científicos se ha venido equiparando al de los llamados intelectuales orgánicos (Gramsci, 2009, p. 1). Es decir, los que organizan la función económica de la clase a la que se encuentran vinculados. La especialización de sus saberes en distintas áreas de conocimiento se ve encauzada hacia la homogenización de los conocimientos y la conceptualización de la realidad amañada a los intereses que pretende defender.

Una vez establecida la situación que amenaza a la objetividad científica, surge la cuestión del poder del discurso, a partir del cual pretenden usarse afirmaciones aparentemente científicas para justificar decisiones basadas en intereses específicos. De ello deriva la trascendencia de analizar algunos discursos políticos que incluyen afirmaciones de diversos intelectuales en temáticas económicas, ambientales y sociales alejadas de la objetividad, que siguen parámetros de manipulación. Para autores como Derrida, el discurso puede ser una forma de dominación, o al menos de convencimiento de las masas para establecer sus prioridades. Algunos ejemplos sobre este aspecto se analizan enseguida.

Uno de ellos es el discurso de campaña del ex presidente Donald Trump, con su plan denominado "The America First Energy Plan", en el cual la base del desarrollo para Estados Unidos sería la independencia energética, a través de la reactivación de la industria del carbón y la expansión de la producción nacional de combustibles fósiles. Además, la defensa del fracking como una técnica limpia y la cancelación de las iniciativas del gobierno anterior para atender a la problemática del cambio climático (García et al., 2018). 
Aquel discurso resulta alarmante en el sentido de su peligro potencial para el medioambiente, especialmente si se atiende a las implicaciones negativas del fracking para recursos como el agua, dado que los escapes de químicos usados para la penetración del agua en las grietas y extracción el recurso, pueden contaminar grandes concentraciones de agua. Asimismo, ha sido criticado por la pretensión de uso del carbón, cuyo efecto en la producción de GEI afecta la capa de ozono.

En el balance del gobierno de Trump se ha identificado un distanciamiento entre su discurso y las situaciones que se presentaron, puesto que Estados Unidos de América ha reducido sus emisiones de GEI y grandes empresas carboneras han cerrado, por ejemplo, Peabody, que produce aproximadamente 170 millones de toneladas anuales de carbón, Arch Coal y Alpha Natural Resources.

Estas situaciones permiten postular algunas condiciones de posibilidad para el fenómeno en la industria del carbón, la primera serían las exigencias internacionales y la presión mediática para sustituir el carbón por opciones de energía limpia o no convencionales. La segunda podría ser el cambio en la dinámica del mercado, dado que la demanda de carbón ha disminuido y los precios de exportación cayeron (de 9,5\% en 2019, en relación con 2018). Adicionalmente, en su informe Perspectivas de energía a corto plazo (2019), la Administración de Información de Energía de EE. UU. "proyecta que las exportaciones de carbón se reducirán en un $16,9 \%$ y el consumo disminuirá un $14,3 \%$ ".

Las cuestiones presentadas dan cuenta de una situación interesante en la dinámica entre el discurso del desarrollo presentado desde el ejecutivo y la realidad que se enfrenta a nivel global. Se inicia por analizar que el discurso basado en el crecimiento económico de EE. UU. por la reactivación de la industria del carbón y la propuesta de autonomía energética fue un componente central para la elección del presidente del país. Sin embargo, al cotejar el discurso con los movimientos del mercado y la tendencia hacia energías limpias se ve una dificultad para el cumplimiento de las promesas presidenciales.
En este punto es necesario incorporar el papel de los intelectuales norteamericanos en la adopción de estas decisiones de apoyo a ciertos sectores energéticos. Un ejemplo claro es la iniciativa Global warming petition cuyo sitio web oficial indica que cerca de 31000 científicos americanos (informando sus grados académicos y participación en la ciencia) han firmado para que se rechacen los acuerdos internacionales en materia de cambio climático, por considerarlos obstáculos para el avance de la ciencia y la tecnología. Además, indican que los GEI y en general el cambio climático no representan peligro para la salud humana. Este último aspecto va en contra de lo indicado en 2018 por la Organización Mundial de la Salud (oms), cuando indicó, entre otros peligros para la salud, los siguientes:

Las temperaturas extremas del aire contribuyen directamente a las defunciones por enfermedades cardiovasculares y respiratorias, sobre todo, entre las personas de edad avanzada. En la ola de calor que sufrió Europa en el verano de 2003, por ejemplo, se registró un exceso de mortalidad cifrado en 70000 defunciones.

Las temperaturas altas provocan, además, un aumento de los niveles de ozono y de otros contaminantes del aire que agravan las enfermedades cardiovasculares y respiratorias.

Las condiciones climáticas tienen gran influencia en las enfermedades transmitidas por el agua o por los insectos, caracoles y otros animales de sangre fría.

El paludismo depende mucho del clima. Transmitido por mosquitos del género Anopheles, el paludismo mata a casi 600000 personas cada año, sobre todo niños africanos menores de cinco años. Los mosquitos del género Aedes, vector del dengue, son también muy sensibles a las condiciones climáticas. (oms, 2018)

En relación con esta contradicción entre posturas de diversos intelectuales, surge la inquietud de la división y orientación de los estudios realizados para concluir aspectos diametralmente diferentes. Además, mientras los movimientos contra las acciones de mitigación o acuerdos para hacer frente al cambio climático (dada la consideración de su inexistencia) fueron centrales para apoyar 
iniciativas como la cancelación de estrategias y planes de preservación ambiental; las dinámicas de mercado se ven encaminadas hacia el otro extremo, que indica que deben tomarse medidas contra el avance del cambio climático, por ejemplo, reducir la demanda de carbón y buscar sustituir formas de energía tradicionales por no convencionales.

El Plan de Desarrollo Territorial para México, para 2019-2024, menciona específicamente en los ejes generales que el desarrollo económico se busca a través del uso eficiente y responsable de los recursos. Sin embargo, algunos megaproyectos contrarían estas afirmaciones discursivas en favor del crecimiento económico, puesto que incorporan estrategias denominadas ecocidios por los medios de comunicación y algunas asociaciones ecologistas. Se trata de cerca de 134 proyectos de minería, 70 en materia petrolera, 50 hidroeléctricos y 15 gaseoductos. Resultan especialmente preocupantes el tren maya, que pasará por dos grandes reservas naturales ${ }^{1}$; la construcción de nuevas refinerías y rehabilitación de otras ${ }^{2}$; por último, el aeropuerto de Santa Lucía ${ }^{3}$.

Es importante mencionar que en los proyectos anunciados por el presidente López Obrador, se encuentran, de forma prioritaria, veinticinco propios de diferentes áreas, desde aumentos pensionales, coberturas de Internet y modernización de puertos y caminos, hasta los que atañen al tema de estudio, los relacionados con el medioambiente de forma directa. Tal es el caso de la iniciativa de

1 Por ejemplo, (1) la reserva de la biósfera de Calakmul, protegida por la onu desde 1989, hogar de múltiples especies (aves, reptiles, anfibios, mariposas y plantas); (2) la reserva de la biósfera de Sian Káan, el área más grande protegida del Caribe, donde habitan más de cien especies de animales y más de mil especies de plantas.

2 Este megaproyecto cuenta con una denuncia ante la Agencia de Seguridad Energía y Ambiente, por la ong Centro Mexicano de Derecho Ambiental, por el desmonte ilegal de manglares. También es importante tener en cuenta los compromisos del Estado frente a la disminución de gases contaminantes y abandonar la dependencia de los combustibles fósiles.

3 Denominado en pluralidad de medios de comunicación como ecocidio. plantación de árboles maderables y frutales, rehabilitación de plantas fertilizantes, créditos ganaderos, modernización de refinerías y construcción de una nueva, producción de más energía eléctrica y modificación de la regulación de la minería. Al tener cierta claridad sobre las temáticas de los proyectos prioritarios, es necesario analizar algunos de estos, especialmente, los que van de la mano o en contravía al discurso de desarrollo propuesto al inicio del periodo presidencial.

La modernización de la producción de combustibles fósiles dista tanto de la idea de uso sustentable de los recursos naturales como de los compromisos que ha adquirido el Estado en cuestión de disminución de las emisiones de GEI. Al estudiar y proponer la generación de energía por medio de las hidroeléctricas, deben también analizarse los efectos futuros del uso inadecuado de estrategias para desarrollar este tipo de energía. Los efectos adversos que estas producen en el medioambiente pasan por afectaciones al ecosistema fluvial, alteración al sistema de caudales, aumentos de la inundabilidad de terrenos aledaños, hasta procesos erosivos y geológicos y algunos otros efectos sobre la flora y la fauna de los lugares donde este tipo de procesos se realiza.

Frente a la construcción del aeropuerto internacional, inicialmente, en Texcoco, ahora proyectado en Santa Lucía, puede verse el papel de los científicos o expertos en la temática ambiental y los efectos que un aval de su parte puede traer en materia ambiental, social y económica. En 2014, se aprobó la Manifestación de impacto ambiental (MIA) del Nuevo Aeropuerto Internacional de la Ciudad de México (Naim), por parte de Juan José Guerra Abud, entonces titular de la Semarnat. En este documento, se avaló la construcción del Naim, sin considerar los efectos reales, como los que sufrió el lago Nabor Carrillo.

Ahora bien, esta manifestación y este aval de los expertos dio lugar a obstáculos importantes para continuar con el megaproyecto, por cuestiones económicas de sobrecostos y ambientales de mayor afectación a la zona e imposibilidad de restauración del daño. Ante estos obstáculos, se presentó la opción de iniciar otro megaproyecto para la construcción del aeropuerto en otro punto, cuya MIA 
resultafavorabledemomento, peronopermiteobviar el hecho de que el daño ambiental ya se hizo, y ahora la inversión económica será mayor.

En materia de desastres industriales que afectan directamente al medioambiente, los ejemplos resultan incluso más claros, como en el desastre de Seveso, al norte de Milán, Italia. La emisión de una nube tóxica de dioxina contó al inicio de la emergencia con opiniones expertas que, ancladas a los andamiajes del poder en turno, insistieron en la ausencia de peligro real para la vida humana, al punto de desplegar las primeras medidas de protección a la población después de cuatro días de inhalaciones de esa toxina (Legadec, 1983).

En el caso de intereses empresariales defendidos y justificados con estudios de apariencia científica, se encuentran los informes de laboratorio alterados y certificaciones falsas presentadas por la compañía norteamericana Saybolt Inc., en los que, violando la norma sobre contaminación del aire, indicaron que la gasolina reformulada cumplía con los parámetros de calidad del aire exigidos. Tal alteración implicó para la empresa pagar una multa de 3,4 millones de dólares.

También se encuentra el caso de los científicos que alteran los informes sobre los efectos en la flora y fauna acuática, causados por los fosfatos usados como aditivos en la industria de detergentes. Especialmente en 1991, cuando se presentaron cargos contra algunos laboratorios por la alteración de estos informes. Por último, es importante mencionar la acusación contra la compañía Monsanto por parte del US Consumer Policy Institute por

haber suprimido una publicación que arroja dudas sobre los efectos de una hormona bovina, la somatotropina, que es manufacturada mediante ingeniería genética y produce un incremento del rendimiento lechero de un $15 \%$. El trabajo publicado finalmente en Nature por Erik Millstone y col. de la Science Policy Research Unit de la Universidad de Sussex, el 20 de octubre de 1994, indica que la hormona puede producir mastitis en las vacas, lo que puede, a su vez, provocar la inclusión de pus en la leche. Monsanto intentó que no se publicara el trabajo, porque se usaron datos de la empresa y acusaron de plagiarios a los autores. (Schulz y Katime, 2003, p. 71)
Los ejemplos plantean un panorama donde pueden hallarse opiniones expertas para defender cualquier iniciativa, proyecto o decisión del gobierno. De esta forma, pueden evidenciarse varios aspectos. El primero es la toma de decisiones que afectan a las poblaciones, los recursos naturales y el futuro mundial bajo criterios económicos. Esta cuestión pudo observarse a partir de alteraciones de documentos e informes científicos para evitar situaciones como el aumento en costos de producción o el retiro de productos del mercado. También se han utilizado estas opiniones científicas para convencer al electorado con la promesa de mejora en el crecimiento económico de los países, dejando fuera factores trascendentales como la protección del tejido social y la conservación medioambiental.

En segundo lugar, el poder del discurso del desarrollo bajo criterios científicos consigue la aprobación de las masas, la aceptación acrítica de proyectos y decisiones que afectarán el bienestar de esta generación y las futuras. Por último, se colige que el papel de los intelectuales es ser herramientas de sustento amañado, para sostener las estructuras de poder -empresas o gobiernos-, encauzando estudios, sesgando muestras y maquillando resultados.

Pese a lo dicho, este análisis no puede generalizarse apresuradamente, puesto que estos fraudes se han descubierto, precisamente, por nuevos estudios y trabajos objetivos, los cuales pueden considerarse realmente científicos, dada la esencia de la ciencia como búsqueda de la verdad. Esto no quiere decir que no se haga presente la problemática del papel de los intelectuales orgánicos, toda vez que, cuando se descubren y desenmascaran los estudios, opiniones e informes falsos pueden haber transcurrido años y ocurrido daños irreparables.

\section{Deconstrucción del discurso del desarrollo y análisis de alternativas al desarrollo con base en la sustentabilidad}

Antes de continuar con el concepto de sustentabilidad, es necesario retomar la base sobre la cual 
se erige la preocupación por los recursos naturales, la supervivencia humana vinculada a ellos y el papel del desarrollo, equiparado con el crecimiento económico. La modernidad ha sido blanco de múltiples críticas desde diferentes sectores, que amalgaman la modernidad con muerte, despojo y la depredación de los recursos y las personas, con fines utilitarios (Berman, 1989, p. 52). Se considera que la modernidad ha traído graves consecuencias para entorno, como el deterioro ambiental, la codicia cuantitativa y legitimación de la destrucción en aras del progreso.

La postura crítica encuentra su contrario en aquellos que relacionan la modernidad con avance social, con una época de desarrollos maravillosos de tecnología y ciencia, además de la superioridad de la razón sobre la superstición, la libertad como estandarte y la democracia. La creación de un mundo sin fronteras gracias a las hazañas tecnológicas que permiten la conectividad al instante y la vida en condiciones de comodidad que antes no eran posibles, son las defensas ante las corrientes prey posmodernas (Lindsay y Pluckrose, 2017 p. 1).

En este punto es relevante mencionar que las posturas premodernas, modernas y posmodernas han querido reducirse a aspectos de izquierdas o derechas políticas, planteando especialmente que la izquierda actual va de la mano con concepciones posmodernas que rechazan los males que entorpecen a la modernidad. No obstante, si se acude a teóricos como Gudynas, en su estudio sobre el desarrollo, se encuentra que gobiernos como el venezolano de Hugo Chávez, el boliviano de Evo Morales, el brasileño de Luis I. Lula da Silva y el ecuatoriano de Correa no escapan a aquello que repelen discursivamente. Su concepto de desarrollo se basa en travestir o enmascarar el crecimiento económico basado en intervención estatal, sin incluir tampoco la discusión de la racionalidad del desarrollo como crecimiento económico o la apropiación de la naturaleza.

En otras palabras, podría afirmarse que no cambian los aspectos criticados de los modelos neoliberales frente aspectos como los recursos naturales, sino que proponen una redistribución de ganancias y un neoextractivismo basado en la intervención constante del Estado. Se presentan mejoras de la concepción social del desarrollo, precisamente por las ideas de redistribución, pero se considera un camino hacia la degradación ambiental, la ausencia de políticas reales y claras de alternativas al desarrollo, como se ha concebido desde la modernidad.

En el caso de las posturas de derecha, el discurso se encamina a la universalización de corte europeo, que enlaza el concepto desarrollo con el crecimiento económico, los fines utilitaristas para un futuro mejor y el progreso anclado a las relaciones de poder. En Más allá del desarrollo, Gudynas (2011) indica que la idea de progreso reforzó la herencia colonial de apropiación de enormes espacios territoriales, con fines extractivistas.

Frente a este aspecto surge la postura de Armando Bartra (2016, p. 34), quien expuso tres razones para no considerar el "mal" (refiriéndose a la modernidad) justificado bajo la idea de progreso. La primera razón esbozada es ética, al considerar que se trivializa la violencia contra la dignidad de los individuos, cuando se despojan y utilizan como medios y no como fines en sí mismos. La segunda es filosófica, pues rechaza el carácter lineal de la historia que, en su postura, la modernidad defiende, para considerar necesarios los episodios técnicos, económicos y sociales. Por último, la razón histórica indica que el despojo es la herramienta de la modernidad para la acumulación de capital.

Antes de continuar con el estudio de las posturas en favor y en contra de la modernidad y su concepción de progreso, es necesario traer a colación lo que se entiende hoy por desarrollo, definido por el doctor Lutz Keferstein (2004), en los siguientes términos:

Desarrollo y crecimiento, se puede comprobar con solo abrir un diccionario, se encuentran concatenados en las concepciones que el humano occidental tiene de dichas nociones. Algo se desarrolla solo si crece. Aunadamente, es probable que, debido a la manera en que se ha traducido el proyecto de la modernidad y la transformación del mundo a lo largo de los dos últimos siglos, no sea tan discutido el afirmar que las ideas derivadas que se tienen frente al vocablo desarrollo cuentan con fuertes connotaciones pragmáticas. Así, al hablar de desarrollo, se 
entiende el crecimiento de aquello que realiza nuestros fines prácticos urgentes y necesarios. (pp. 1-9)

Establecidas de manera general las posturas en favor y en contra de la idea del progreso a partir de la modernidad, surgen los grandes cuestionamientos al discurso del desarrollo que ha venido gestándose desde cualquier extremo político y contando con el sustento de pluralidad de intelectuales orgánicos. Los cuestionamientos se refieren, por un lado, a un ataque al componente ideológico que sostiene las estrategias de mercado, los hábitos consumistas y el uso desmedido de los recursos naturales. Desde finales de 1980, se han presentado diversas posturas que rechazan la idea del desarrollo basado solo en la acumulación de capital. La tendencia capitalista ha permeado en los hábitos de las personas y promueve la defensa de los modelos consumistas para no perder el estilo de vida que se lleva. Esta situación ha coadyuvado al mantenimiento de una posición individualista que desconoce la otredad y no incorpora la preservación de recursos para generaciones futuras.

Por otro lado, también ataques a la idea de desarrollo de la modernidad se refieren a un paradigma considerado neomalthusiano, por los defensores de la modernidad y del crecimiento infinito por avance tecnológico y científico. Este paradigma señala la ley límite de la naturaleza, que indica que en algún punto el planeta no soportará mayor crecimiento demográfico, la carga excesiva a los ecosistemas y tampoco soportará la degradación ambiental, especialmente en lo que refiere a recursos hídricos. De acuerdo con estas posturas, surgen algunas construcciones teóricas, técnicas y optimistas sobre alternativas al desarrollo, iniciando por la sustentabilidad.

Para abordar la temática, es importante partir de la etimología de sustentable que se une al concepto de desarrollo (planteado en líneas anteriores). Sustentabilidad viene del verbo latino sustinere, cuyo significado es mantener o sustentar, que busca mantener las condiciones del entorno ecológico para la supervivencia humana.

Ahora bien, para lograr la aplicación eficiente del concepto de sustentabilidad y sus elementos, conviene aclarar que, a grandes rasgos, lo que pretende incluirse en el concepto de desarrollo son los factores socioambientales. Se trata de buscar un equilibrio que permita el avance de la humanidad sin que ello le cueste su supervivencia.

La tabla 1 presenta explica las diferentes estrategias internacionales creadas con miras a preservar el medioambiente atendiendo a criterios de sustentabilidad incorporada al desarrollo. La finalidad es evidenciar que no son pocos los instrumentos y el avance en términos de movimientos internacionales para frenar la degradación ambiental.

Tabla 1. Estrategias e instrumentos internacionales para preservar el medioambiente con criterios de sostenibilidad

\begin{tabular}{|l|l|l|}
\hline Instrumento & 1972 & $\begin{array}{l}\text { Protección ambiental con visión antropocéntrica } \\
\text { Estrategias de acción para el medioambiente sano: evaluación y gestión }\end{array}$ \\
\hline $\begin{array}{l}\text { Conferencia de la Organización } \\
\text { de Naciones Unidas sobre } \\
\text { medioambiente humano }\end{array}$ & 1972 & $\begin{array}{l}\text { Temas tratados: } \\
\text { - Incremento demográfico } \\
\text { - Crecimiento industrial } \\
\text { - Recursos naturales } \\
\text { - Contaminación }\end{array}$ \\
\hline Club de Roma & 1987 & $\begin{array}{l}\text { Incorporó el termino sustentabilidad y enfocó } \\
\text { esfuerzos hacia la máxima del futuro común. }\end{array}$ \\
\hline $\begin{array}{l}\text { Comisión Mundial sobre el ambiente: } \\
\text { Informe de Brundtland }\end{array}$ & 1992 & $\begin{array}{l}\text { Los acuerdos y temas discutidos se basan en } \\
\text { - Proteger el medioambiente y la biodiversidad. } \\
\text { - Consagrar y definir el derecho al desarrollo, } \\
\text { bajo parámetros de solidaridad y sostenibilidad. }\end{array}$ \\
\hline $\begin{array}{l}\text { Conferencia de las Naciones Unidas } \\
\text { sobre medioambiente y desarrollo } \\
\text { (Conferencia de Río de Janeiro) }\end{array}$ & \multicolumn{2}{|c|}{ - } \\
\hline
\end{tabular}




\begin{tabular}{|c|c|c|}
\hline Agenda 21 & 1992 & $\begin{array}{l}\text { Contiene } 27 \text { principios; es considerada soft law y consagra aspectos importantes en } \\
\text { cuanto a disposición de recursos y acciones adecuadas para la conservación. }\end{array}$ \\
\hline $\begin{array}{l}\text { Primera Conferencia de Ciudades } \\
\text { Europeas Sostenibles }\end{array}$ & 1994 & $\begin{array}{l}\text { Trató temas como la sostenibilidad, justicia social, participación ciudadana en } \\
\text { decisiones locales. }\end{array}$ \\
\hline Protocolo de Kioto & 1997 & $\begin{array}{l}\text { Entró en vigor en 2005, sobre temas como la transacción de emisiones e } \\
\text { implementación cooperativa y para llegar al desarrollo limpio. }\end{array}$ \\
\hline Convención de Aarhus & 1998 & Se basa en el acceso a la justicia ambiental y participación ciudadana. \\
\hline $\begin{array}{l}\text { Cumbre Mundial por el Desarrollo } \\
\text { Sostenible (Río+10) }\end{array}$ & 2002 & $\begin{array}{l}\text { Se llevó a cabo en Johannesburgo, para tratar especialmente el tema de las } \\
\text { responsabilidades comunes pero diferenciadas. }\end{array}$ \\
\hline $\begin{array}{l}\text { IV Informe del Grupo } \\
\text { Intergubernamental de Expertos } \\
\text { sobre Cambio Climático }\end{array}$ & 2007 & $\begin{array}{l}\text { El Informe del IPCC presentó resultados prudentes pero claros con respecto a las } \\
\text { proyecciones del cambio climático. }\end{array}$ \\
\hline $\begin{array}{l}\text { Conferencia de las Naciones Unidas } \\
\text { para el desarrollo sostenible (Río+20) }\end{array}$ & 2009 & $\begin{array}{l}\text { Trató de economía verde, marco institucional de desarrollo sostenible y el global } \\
\text { green new deal: eficiencia energética, entre otros. }\end{array}$ \\
\hline Acuerdo de París & 2015 & $\begin{array}{l}\text { Se basó en los retos del cambio climático y la urgencia de adoptar medidas } \\
\text { colaborativas. }\end{array}$ \\
\hline $\begin{array}{l}\text { 24. ' Conferencia de las partes (COP } \\
\text { 24) de la Convención Marco de las } \\
\text { Naciones Unidas sobre Cambio } \\
\text { Climático (Katowice, Polonia) }\end{array}$ & 2018 & $\begin{array}{l}\text { Se reunieron las partes firmantes del Protocolo de Kioto y conmemoraron el } \\
\text { Acuerdo de París, por lo cual las temáticas giraron en torno a las acciones en } \\
\text { materia de regulación, para hacer posible el cumplimiento de los compromisos. }\end{array}$ \\
\hline
\end{tabular}

Pese a estos esfuerzos de nivel global, los resultados no han generado el impacto necesario para frenar la degradación ambiental, la pérdida de biodiversidad y el cambio climático. Por el contrario, la Tierra enfrenta sus puntos más altos de temperatura, según señalan estudios propios de la materia, que pueden contrastarse con los que manifiestan que el cambio climático es una variación propia de los ciclos terrestres y las causas antropogénicas no son determinantes. En concordancia con los primeros, atendiendo criterios de prudencia, se suscriben las manifestaciones del IPCC como grupo intergubernamental que analiza estos fenómenos de variación climática. En estas manifestaciones se indica que, si bien la tierra experimenta naturalmente estos ciclos, la actividad humana ha tenido incidencia en la temporalidad entre ciclos y en el aumento de algunos efectos de las variaciones, especialmente, por la acumulación de gases en la atmosfera.

La amenaza del aumento de los efectos del cambio climático y la reducción de los recursos naturales envuelve a la humanidad en lo que Ulrich Beck (1998) ha denominado "sociedad del riesgo".
Este riesgo se amplifica por el avance abrumador del consumismo depredador, que aumenta la producción de residuos sólidos, la presencia de plásticos de un solo uso en el océano y los ríos; además del uso de los combustibles fósiles que aumentan las emisiones de GEI. En este último aspecto, algunos estudios analizan el efecto de estas concentraciones de $\mathrm{CO}_{2}$ en el ambiente como beneficiosas para algunas especies de plantas, no obstante, no se puede recurrir a este argumento para justificar las emisiones, dado que se pone en peligro la salud humana, por baja calidad del aire.

La racionalidad ambiental ${ }^{4}$ se encamina hacia una reasignación del mundo, con base en la ley límite de la naturaleza. Se trata de un cambio que incorpore el saber ambiental al campo subjetivo de las personas, como materia prima para la construcción de la sustentabilidad.

4 Entiéndase como racionalidad ambiental aquella que pretende traducirse a nivel normativo y social. Es decir, la que resulte de una acción explicable y aceptable a nivel social y, de paso, en comportamientos sociales previsibles, dado que proviene precisamente de la previsibilidad. 
Justamente, esa materia es "el diseño de nuevos mundos de vida, cambiando el sentido de los signos que han fijado los significados de las cosas" (Leff, 2004, p. xii).

Leff (2020) ha dedicado sus obras a la deconstrucción del concepto desarrollo, para construir un nuevo concepto, basado en la apropiación de los individuos de la naturaleza no como mercancía. Para lograr esta reconstrucción del mundo, los intelectuales no pueden ser orgánicos, deben estar al servicio de la verdad y contar con una conciencia ecológica. Asimismo, ha incorporado una crítica importante hacia la racionalidad técnico-económica que ha invisibilizado las condiciones de visa que propugnan por un "mundo humano más sensible, más digno y justo" (p. 16).

A fin de comprender la manera como se lleva a cabo el cambio hacia la racionalidad ambiental, se trae a colación lo señalado por Leff $(2004$, p. 215$)$ al respecto de los cuatro niveles de racionalidad. La racionalidad material, como nivel inicial, que pretende establecer un sistema de valores que guíen los comportamientos sociales, incluyendo los saberes ambientales y principios éticos de la sustentabilidad. Seguida por una racionalidad teórica que da soporte a la construcción de una racionalidad productiva diferente de la actual, con elementos como el potencial ecológico y las aportaciones culturales de cada comunidad. La racionalidad técnica o instrumental, como siguiente nivel, es la que produce aquellos vínculos funcionales entre objetivos sociales y bases materiales del desarrollo, este aspecto incluye sistemas tecnológicos adecuados. Finalmente, la racionalidad cultural permite incluir las identidades diferenciadas de culturas diversas y reconoce su singularidad.

Ante la existencia de iniciativas de cooperación internacional, la tecnología verde ${ }^{5}$ o limpia y la economía azul ${ }^{6}$ que ofrecen alternativas que

5 Tecnología verde es el uso de elementos de la naturaleza de manera sostenible, para sustituir, con procesos basados en la sustentabilidad de la producción, elementos que contaminantes y nocivos para el medioambiente.

6 Se refiere a la mejora y actualización continua de las técnicas y tecnologías de producción, para hacerlas ambientalmente amigables y no solo re- no implican parar las industrias o volver a los modos de vida de los antepasados - idea que resulta poco probable por el modo de vida actual y la dependencia tecnológica-, es evidente que lo que falta para completar estos procesos y aplicar las alternativas para la preservación ambiental es buscar la restauración de la relación del ser humano con la naturaleza.

La forma como logra articularse es la imprescindible aplicación de la ecología política, entendida como la comprensión de los problemas ambientales a partir de su trasfondo político. Es decir, explicaciones que conectan circunstancias sociales y económicas que requieren implementar políticas con fundamentos en la sustentabilidad, para comprender sus causas y encontrar soluciones acordes con la preservación de los recursos naturales.

La ecología política aplicada en todos los escenarios de decisión del poder produce acciones y políticas en favor del medioambiente. Además, permite pensar en la otredad, producto de la racionalidad ambiental que incluye la diversidad cultural. Esto se logra con una visión biocéntrica, que no relegue la naturaleza al papel de mercancía, sino que posicione al hombre en un sistema complejo que incluye más elementos que deben preservarse.

Adicionalmente, se propone incluir un despliegue tecnológico limpio y la reformulación de relaciones de poder en escenarios globales, que permita una participación igualitaria o siquiera representativa, en las decisiones que competen a la humanidad. No se busca dejar de lado el crecimiento económico, sino replantear su forma de desenvolverse, al ampliar el concepto de desarrollo, incluyendo otros factores determinantes, como los sociales y los ambientales. Tal es la propuesta de la economía ecológica, planteada como alternativa a la economía ambiental, cuyo objetivo es "considerar la existencia física de los objetos económicos, en forma de recursos, antes de ser valorados, y de su existencia posterior, en forma de residuos" (Rincón, 2020, p. 29).

emplazar productos sintéticos por naturales, toda vez que ello traería consigo una crisis de extinción de especies vegetales o su sobreexplotación. 


\section{Conclusiones}

A partir de la investigación, se concluye que ha existido y, actualmente, se ha amplificado un discurso del concepto de desarrollo, limitado, en principio, a la esfera económica, lo que obstaculiza la inclusión de los factores diversos que intervienen en el avance social. Se dejó de lado la idea de desarrollo como perfeccionamiento humano, que buscaba, principalmente, la armonía de la vida en colectivo con el desarrollo de las sociedades, centradas en una visión amplia, no individualista. Esta afirmación no implica una glorificación de las sociedades pasadas y su visión de desarrollo, por el contrario, busca extraer los elementos importantes de la concepción del progreso como armonía del ser humano y su camino hacia la mejora constante.

Si bien se advierte el abandono de la concepción tradicional de desarrollo o progreso, también se rescata la importancia de los movimientos contrahegemónicos y su visión contraria de la naturaleza, fuera del paradigma utilitarista, perpetuado con el capitalismo y la colonización. En América Latina, principalmente, se ha buscado generar contrapesos a la visión mercantilista de los recursos naturales, a través de iniciativas como el "buen vivir" y la visión biocéntrica del ser humano. Es decir, su ubicación dentro de un entorno sin el cual su garantía de supervivencia se ve gravemente amenazada. Estas estrategias son incipientes y se ven obstaculizadas por posturas de negación de fenómenos que evidencian degradación ambiental, como el calentamiento global o el riesgo de estrés hídrico.

Se concluye que, sin un cambio del concepto de desarrollo y su adecuación a las circunstancias ambientales actuales y las previsiones a futuro, se pondría en riesgo incluso la libertad de consumo que defiende el neoliberalismo. Esto debido a que, sin materias primas ni recursos para la elaboración de los productos de consumo masivo, peligra la economía mundial; sin mencionar las implicaciones a la salud, la calidad de vida y la supervivencia.

Asimismo, queda claro el papel central de intelectuales orgánicos en la prolongación del discurso del desarrollo, equiparado con crecimiento económico, y su legitimación hacia las masas, separándose de la objetividad y la autonomía, debido a la pertenencia a los andamiajes del poder. Los intereses económicos han desviado la labor que deben desempeñar los intelectuales, pues la ciencia va en busca de la verdad y se cimienta en la objetividad, para acercar al ser humano hacia un progreso en el saber y comprensión del mundo que lo rodea.

El rol central del intelectual orgánico, como legitimador de acciones del poder, puede prolongar este sistema depredador de uso indiscriminado de recursos naturales y convertir su labor en una máscara de la destrucción. Esta cuestión se ejemplifica a través del mito de Fausto de Goethe, en el cual el personaje principal cree escuchar las palas construyendo su anhelado dique, mientras estas estaban cavando su tumba. Esto resulta una fiel representación de la humanidad cuando destruye la naturaleza y no advierte que está construyendo el camino hacia su propia destrucción, con un sentimiento impersonal y de origen económico.

En un mundo convulso y en permanente riesgo, surge la necesidad de buscar nuevas alternativas a este discurso del desarrollo, a través de una vida sustentable, que amalgame los saberes ancestrales de preservar el entorno, vivir en armonía con la naturaleza y mantener una relación equilibrada entre el ser humano y el medioambiente, junto con los avances tecnológicos e industriales basados en energías renovables, verdes o limpias. Es indispensable repensar el progreso, aceptar la complejidad de su deconstrucción y la ampliación de su concepto a las circunstancias reales de la humanidad, proponiendo alternativas al desarrollo, sin reducir la labor a encuentros mundiales en los cuales media la voluntad de las potencias económicas y quedan fuera de la discusión los temas incómodos para el sistema capitalista.

Para materializar los cambios en el concepto y en el discurso del desarrollo, se requiere una sociedad activa, consciente ecológicamente y participativa de las decisiones, proyectos e iniciativas de sus Gobiernos, en escenarios nacionales e internacionales. Se demanda una sociedad atenta a las luchas sociales por su entorno y bienestar actual y futuro, puesto que "estos movimientos posibilitan horizontes emancipación y señalan trazos 
de cambio tanto en la sociedad como en el derecho" (Anglés-Hernández et al., 2020, p. 233). Es indispensable una sociedad formada en la racionalidad ambiental, con una visión biocéntrica, alejada del consumismo.

\section{Referencias}

Anglés-Hernández, M. García, E. y López A. (2020). Racionalidad legal, consulta ambiental y lucha de los pueblos indígenas por el desarrollo propio. En R. Gutiérrez y M. Burgos (Coord.). Globalización, neoliberalismo y derechos de los pueblos indígenas en México. (pp. 209237). Instituto de Investigaciones Jurídicas, Unam.

Bartra, A. (2016). Goethe y el despojo. Ítaca.

Beck, U. (1998) La sociedad del riesgo. Hacia una nueva modernidad (Trad. J. Navarro). Paidós.

Berman, M. (1982). Todo lo sólido se desvanece en el aire. La experiencia de la modernidad. Siglo Veintiuno.

Brook, A. y Stainton, R. (2000). Knowledge and Mind. A Philosophical Introduction. Bredford book.

Declaración de Cocoyoc (1978). La dimensión ambiental en las políticas y planes de desarrollo. Centro Interamericano de Formación en Ciencias Ambientales.

Esteva, G. (2000). Desarrollo. En A. Viola (Ed.). Teoría y estudios etnográficos en América Latina (pp. 67-101). Paidós.

García, V., Lucatello, S., Quintana, F., Delgado, G., Gómez, E., Navajas, T., Rueda, J. y Alarcón, F. (2018). Del oasis al desierto. La política anticlimática de Donald Trump. Unam.

Gramsci, A., (2009). La formación de los intelectuales. Disponible en http://juventud.psuv.org.ve/wp-content/uploads/2009/05/la-formacion-de-los-intelectuales-gramsci.pdf

Gudynas, E. (2011) Debates sobre el desarrollo y sus alternativas en América Latina: una breve guía heterodoxa. En M. Lang y D. Mokrani (Eds.). Más allá del desarrollo. (pp. 21-53). Fundación Rosa Luxemburgo, AbyaYala.

Habermas, J. (1990). Conocimiento e interés. Taurus.

Hubert, A. (2004). El enigma del hombre según Anselmo de Canterbury. Teología y Vida, 45(4), 494-530. http:// dx.doi.org/10.4067/S0049-34492004000400002

Kant, I. (2006). Ideas para una historia universal en clave cosmopolita. Unam. (Primera edición de 1784).

Keferstein, L. (2005). Keferstein, L. (2005) The Kantian Cosmopolitan Intent and the xxi Century Neoliberalism. Revista Jurípolis, 3(1), 205-215.

Keferstein, L. (2004). Educación y desarrollo en el sexenio de la muerte filosófica. Revista Electrónica del IPN: $\mathrm{Hu}$ manidades, tecnología y ciencia, 2(11), 1-9.
Leff, E. (2004) Racionalidad ambiental, la reapropiación social de la naturaleza. Siglo Veintiuno.

Leff, E. (2006). La ecología política en América latina. En H. Alimonda, C. Toro y F. Martín (Eds.). Los tormentos de la materia. Aportes para una ecología política latinoamericana (pp. 129-166). Clacso.

Leff, E. (2020). Racionalidad ambiental. Aprendiendo a vivir en las condiciones de la vida. En E. Leff, H. Castro y J. Carrizosa (Eds.). Viraje hacia la vida: debates $y$ reflexiones en torno a la sustentabilidad (pp. 15-16). Universidad Autónoma de Occidente.

Legadec, P. (1983). La civilización del riesgo. Mapfre.

Lindsay, J. y Pluckrose, H. (2017). Un manifiesto contra los enemigos de la modernidad. Disponible en https://areomagazine.com/2017/08/22/a-manifesto-against-the-enemies-of-modernity/

Maddison, A. (2004). La economía de occidente y la del resto del mundo en el último milenio. Universidad Carlos III.

Marx, K. (2008). Contribución a la crítica de la economía política. Siglo Veintiuno. (Primera edición de 1859).

Murat, E. (2018). Informe sobre la $24^{a}$ Conferencia de las Partes (COP24). Senado de la República.

Nisbet, R. (1979). La idea de progreso. Institute for Humane Studies.

Organización de las Naciones Unidas -ONU. (1987). Report of the World Commission on Enviroment and Development "Our Common Future". http://netzwerk-n.org/ wp-content/uploads/2017/04/0_Brundtland_Report1987-Our_Common_Future.pdf

Ramón, R. (2005). Veritas filia temporis en Averroes. Comentario a Metafísica II. Tópicos, Revista de Filosofía, 29, 13-28.

Rincón, E. (2020). Debate sobre los problemas ambientales desde la ciencia económica: economía ambiental versus economía ecológica. Revista Ethos, 1(1), 15-27.

San Agustín. (2011). La ciudad de Dios (F. Montes de Oca, trad.). Biblioteca de Autores Cristianos. Disponible en https://historicodigital.com/download/la-ciudadde-dios.pdf

Santo Tomás de Aquino. (2001). [Summa Theologiae] Suma de teología (Edición dirigida por los regentes de estudios de las Provincias Dominicanas en España; $4{ }^{\text {a }}$ edición). Biblioteca de Autores Cristianos.

Schulz, P. y Katime, I. (2003). Los fraudes científicos. Revista Iberoamericana de Polímeros, 4(2), 1-90.

Smith, A. (1776). La riqueza de las naciones (C. Rodríguez-Braun, ed.). Titivillus. https://bit.ly/2QsTVAA

Stuart, J. (1984). El utilitarismo. Alianza.

Torres, M. (2008). El derecho de veto en el Consejo de Seguridad de Naciones Unidas. La historia de la válvula de seguridad que paralizó el sistema. ACDI. 